\title{
STRUCTURE OF THE RENAL LYMPH NODE IN EXPERIMENTAL PROSTATE CARCINOGENESIS AND LASER PROSTATECTOMY
}

\author{
Vadim Astashov ${ }^{1 \otimes} \mathbb{( B D}$, Andrey Lomshakov² \\ ${ }^{1}$ Peoples Friendship University of Russia (RUDN University) \\ ${ }^{2}$ LLC MC «Stolitsa», Moscow, Russia \\ vastashov3@gmail.com
}

ABSTRACT - A promising and developing area in oncology is the use of high-energy laser radiation. The structure of the prostate and renal lymph nodes of mice was studied on the $18^{\text {th }}$ day of the experimental prostate tumor and after its instrumental and laser resection. On the $18^{\text {th }}$ day of prostate tumor growth in mice, severe T-zone hyperplasia and sinus reaction were detected in the renal lymph nodes. On the $18^{\text {th }}$ day after instrumental resection of the tumor, $\mathrm{t}$-zone hyperplasia persists, and the transport function of the renal lymph nodes is partially restored. On the $18^{\text {th }}$ day after resection of the tumor with high-energy ND:YAG laser radiation, with a wavelength of $1064 \mathrm{~nm}$, a photostimulating effect was detected in the peripheral heating zone, which is expressed in the restoration of the structure of regional lymph nodes.

KEYW ORDS - experimental prostate tumor, renal lymph nodes, prostatectomy, high-energy laser radiation.

\section{INTRODUCTION}

During carcinogenesis, various stages of the immune response are disrupted, followed by its failure, immunological tolerance is developed, caused by an excess of tumor antigens, activation of suppressor T-lymphocytes and macrophages, and blocking of the effector link of the immunocompetent system by circulating tumor antigens, antibodies, and antigenantibody immune complexes; [1,2]. Radical prostatectomy is the optimal method of treating a malignant prostate tumor and eliminating the immunosuppressive effects of cancer on the body and in the early stages of the disease, when there are no metastases to the lymph nodes. One of the promising and developing areas of Oncology practice is the use of highenergy laser radiation used for dissection of tissues, hemostasis, evaporation of pathological formations of blood serum coagulation with local byalinization of tissues [5]. An important condition for preventing postoperative recurrence and tumor metastasis is the structural and functional restoration of the regional lymphatic apparatus. The study of morphological transformations occurring in regional lymph nodes after resection of an experimental prostate tumor will assess the degree of recovery of their structural and functional organization and the state of the local immune response.

The purpose of our study was to identify structural changes in the regional lymph nodes of the prostate (renal) during its experimental tumor and after prostatectomy using an instrumental method and using high-energy laser radiation.

\section{RESEARCH MATERIAL AND METHODS}

We used sexually Mature 14-week-old male NEA mice in the amount of 40 pieces (vivarium Institute of Cytology and genetics SB RAS, Novosibirsk). All experiments were performed in accordance with the principles of humanity set out in the European community Directive (86/609 / EC). An experimental model of prostate carcinogenesis was created by inoculating (under ether anesthesia) a diluted cell strain of the Ehrlich's transplanted ascitic tumor into the prostate parenchyma $-0.2 \mathrm{ml}$ of ascitic fluid containing 500-550×103 atypical tumor cells [ 4 ]. The animals were divided into 4 groups of 10 individuals: 1 group - intact animals (control); 2 - animals on the $18^{\text {th }}$ day of experimental carcinogenesis; 3 - animals on the $13^{\text {th }}$ day of the experiment, with preliminary complete instrumental resection of the tumor on the $5^{\text {th }}$ day of its growth. $4-$ animals on the $13^{\text {th }}$ day of the experiment, with preliminary full laser resection of the tumor on the $5^{\text {th }}$ day of its growth. Prostatectomy was performed under ether anesthesia in compliance with the methods of asepsis and antiseptics: in the $3^{\text {rd }}$ group by laparoscopic method using a microsurgical instrument, and in the $4^{\text {th }}$ group using a flexible optical fiber emitter from an ND:YAG laser pulse radiation device, with a radiation wavelength of $1064 \mathrm{~nm}$, pulse energy of $100 \mathrm{j} / \mathrm{cm}^{2}$, with a pulse duration of $20 \mathrm{MS}$, and a frequency of $1 \mathrm{~Hz}$. (Laura 50, Lazerus LLC, Russia): After prostatectomy, the material was collected on day 18 after the tumor was inoculated. For histological examination, after euthanasia, the prostate, tumor fragments, and renal lymph nodes were taken. According to the standard histological method, the material was wired, and longitudinal median histological sections were made with a thickness of 5-7 microns, which were stained with hematoxylin-eosin and Azur II-eosin. The preparations were studied using light 
microscopes (Leica (Germany), MBS-10 (Russia) at magnification of 32, 400 and 1000 times. Morphometric of structural and functional zones of lymph nodes and tumor tissue was performed [7]. The results of the experiments were processed using standard methods of variation statistics, and the probability of reliability of differences was determined using the Student's criterion at $\mathrm{p}<0.05$.

\section{RESULTS AND DISCUSSION}

On the $5^{\text {th }}$ day of induced carcinogenesis, a tumor node with a volume of $2 \mathrm{~m} 3$ is formed in the prostate, consisting of polymorphic atypical cells. On the $18^{\text {th }}$ day of prostate carcinogenesis, almost complete replacement of its structural components with these cells was found: the tumor parenchyma was $82.3 \pm 0.63 \%$, the tumor stroma was $17.7 \pm 0.43 \%$, and the stromal - parenchymal index was $0.22 \pm 0.01$. Lymphoplasmocytic infiltration along the course of blood vessels, focal necrosis and hemorrhages were noted. Metastases on the 18th day of carcinogenesis were determined in the marginal and cerebral sinuses of the renal lymph nodes. In the renal lymph nodes on the $18^{\text {th }}$ day of tumor growth, pronounced signs of paracortical hyperplasia were revealed - the T-dependent zone increased by $87 \%$ compared to the $1^{\text {st }}$ group, the size of the brain cords decreased by $14 \%$, and the brain sinuses - by $52 \%$. In General, the size of the cortical substance of the lymph nodes increased by $74 \%$ in comparison with intact animals (control), and the area of the brain substance decreased by $16 \%$ - the cortical-brain index increased by 2.3 times in comparison with the control. The area of the B-dependent zone in the lymph nodes decreased by $10 \%$ compared to intact animals (Table 1).

On the $18^{\text {th }}$ day of tumor growth, when developing a late stage of carcinogenesis, accompanied by metastasis (II-III stage), the redistribution of lymph flow and changing of structural specialization of the lymph nodes revealed marked paracortical hyperplasia determined characteristic, a sinus reaction to the metastasis - reducing the size of the cerebral sinuses, and changes in the structural and functional specialization of lymph nodes and fragmented type to intermediate [3]. On the $13^{\text {th }}$ day after the instrumental prostatectomy (prostatectomy was performed on the $5^{\text {th }}$ day of tumor growth), only single atypical tumor cells were detected in the marginal and cerebral sinuses of the renal lymph nodes. In the lymph nodes after instrumental prostatectomy, compared with the group without removing the tumor, an increase in the size of the medulla and a decrease in the cortical area was revealed. The structural and functional organization of lymph nodes after prostatectomy indicates an increase in their transport potential, which is confirmed by a decrease in the cortical-brain index (Table 1). The structure of lymph nodes revealed a $12 \%$ decrease in the area of secondary and primary lymphoid nodules and paracortical zone (Table 1). It can be assumed that the path of lymph transport within the lymph nodes changes, which is confirmed by a reduction in the size of the marginal sinus and an increase in the area of the brain. On the $13^{\text {th }}$ day after laser prostatectomy (prostatectomy was performed on the $5^{\text {th }}$ day of tumor growth), atypical tumor cells were not detected in the marginal and cerebral sinuses of the renal lymph nodes. In the lymph nodes after laser prostatectomy, compared with the group without removing the tumor, an increase in the size of the medulla and a decrease in the cortical area was also revealed. in comparison with the group with instrumental prostatectomy, these changes are amplified (the size of the cortical substance decreases by $7 \%$, and the brain substance increases by $4 \%$ ). The structural and functional organization of lymph nodes after laser prostatectomy indicates an increase in their transport potential, which is confirmed by a decrease in the cortical-brain index, compared with the group with instrumental prostatectomy, this index decreases by $10 \%$ (Table 1 ) the structure of lymph nodes in comparison with the group without tumor removal, and in contrast to the group with instrumental correction, there was an increase in the area of secondary and primary lymphoid nodules (by 26\% and 54\%), a decrease in the area of the paracortical zone by $45 \%$ 9 , and in comparison with the group with instrumental prostatectomy by $74 \%$. The size of the marginal and cerebral sinuses in the lymph nodes during laser resection increases, in comparison with the group with instrumental resection. The data obtained indicate that the pathway of lymph transport within the lymph nodes changes in comparison with the group with instrumental prostatectomy.

\section{SUMMARY}

The results obtained indicate that on the $18^{\text {th }}$ day of observation, 13 days after instrumental removal of the prostate tumor in mice, structural transformations were detected in the kidney lymph nodes, which indicates activation of their transport function, persistent paracortical hyperplasia, and inhibition Of the b-link of the immune response (reduction of the area of secondary lymphoid nodules and brain cords). $\mathrm{n}$ the renal lymph nodes of mice, after instrumental removal of a prostate tumor, we found only a partial restoration of their structural and functional organization in comparison with intact animals, which indicates that their barrier-filtration function was suppressed after surgery. After laser resection is more complete restora- 
Table 1. Sizes of structural and functional zones of renal lymph nodes in intact animals (control) in experimental prostate tumors on day 18 (EPT), after instrumental prostatectomy (EPT +PE), after laser prostatectomy $(E P T+L P E)(M \pm m) \%$

\begin{tabular}{|c|c|c|c|c|}
\hline $\begin{array}{l}\text { Structural components of renal } \\
\text { lymph nodes }\end{array}$ & Control & EPT & $E P T+P E$ & EPT + LPE \\
\hline Germinative center & $0,98 \pm 0,14$ & $1,88 \pm 0,11$ & $0,91 \pm 0,14$ & $1,44 \pm 0,17 \cdot \cdot$ \\
\hline Corona & $1,3 \pm 0,14$ & $1,9 \pm 0,13$ & $1,18 \pm 0,11$ & $3,33 \pm 0,19$. \\
\hline Secondary lymphoid nodules & $2,28 \pm 0,06$ & $3,78 \pm 0,07^{*}$ & $2,09 \pm 0,1$ & $4,77 \pm 0,07 . \bullet$ \\
\hline Primary lymphoid nodules & $2,34 \pm 0,16$ & $1,53 \pm 0,08^{*}$ & $0,88 \pm 0,07$ & $2,37 \pm 0,23 \cdot \bullet$ \\
\hline Interfollicular zone & $0,76 \pm 0,13$ & $1,32 \pm 0,1$ & $0,76 \pm 0,11$ & $2,93 \pm 0,15 \cdot \bullet$ \\
\hline Paracortical zone & $20,1 \pm 0,62$ & $37,58 \pm 0,65^{*}$ & $33,28 \pm 1,32 \cdot$ & $24,5 \pm 0,78 \cdot \bullet$ \\
\hline Brain cords & $46,67 \pm 2,95$ & $40,33 \pm 0,47$ & $39,55 \pm 2,15$ & $41,86 \pm 2,1$ \\
\hline Brain sinuses & $23,1 \pm 1,56$ & $11,22 \pm 0,36^{*}$ & $20,86 \pm 1,1$ & $21,2 \pm 0,73$ \\
\hline Capsula & $1,9 \pm 0,17$ & $2,47 \pm 0,17^{*}$ & $1,03 \pm 0,09$ & $0,44 \pm 0,12 \cdot$. \\
\hline Marginal sinus & $2,72 \pm 0,19$ & $1,65 \pm 0,12$ & $1,53 \pm 0,09$ & $1,8 \pm 0,17$ \\
\hline Trabeculas & $0,22 \pm 0,08$ & $0,13 \pm 0,06$ & $0,03 \pm 0,02$ & $0,12 \pm 0,06 \cdot \bullet$ \\
\hline Cortical substance & $25,43 \pm 0,4$ & $44,2 \pm 0,9^{*}$ & $37,0 \pm 1,29$ & $34,56 \pm 0,36 \cdot$ \\
\hline Brain substance & $69,73 \pm 1,15$ & $51,55 \pm 0,91^{*}$ & $60,41 \pm 1,62 \cdot$ & $63,08 \pm 0,66$. \\
\hline B-dependent zone & $52,04 \pm 0,98$ & $46,95 \pm 1,1^{*}$ & $43,28 \pm 1,76$ & $51,92 \pm 0,67$. \\
\hline $\mathrm{K} \backslash \mathrm{M}$ index & $0,37 \pm 0,01$ & $0,86 \pm 0,02 *$ & $0,61 \pm 0,02$ & $0,55 \pm 0,02 \cdot \bullet$ \\
\hline
\end{tabular}

* - the differences are significant in comparison with the control at $P<0,05$.

- - differences are significant in comparison with the group on the 18 th day of EPT at $P<0,05$

.- - differences are significant in comparison with the group on the 18th day of EPT $+\angle P E$ at $P<0,05$

tion of structural and functional organization of the renal lymph nodes, resulting in the reduction paracortical hyperplasia, activation of transport function and restore the sizes of b-dependent zones of the lymph nodes. As shown by the data we received earlier [6] in the pelvic lymph nodes of mice after laser prostatectomy, the restoration of structural and functional organization is also observed, and the study of their cell composition indicates pronounced processes of proliferation and blast transformation of lymphoid cells, activation of the macrophage reaction, which reflects the high activity of the local immune response after prostatectomy. Resection of an experimental prostate tumor, regardless of the method, leads to the restoration of the structural organization of the renal lymph nodes, which is probably the result of a decrease in local antigenic stimulation and endogenous intoxication after removal of the tumor.

Laser resection for prostate tumors is more effective in restoring the structural organization of regional lymph nodes to the prostate, which is expressed in normalization of the ratio of T-and B-dependent zones within the lymph nodes and activation of transport function (increase in the size of the marginal and cerebral sinuses). It can be assumed that high-energy laser radiation in the IR region of the spectrum during local thermal photodestruction of a prostate tumor has a local photostimulating effect in the peripheral heating zone on interstitial spaces, lymphatic capillaries and vessels, and lymph nodes due to absorption and scattering of radiation.

Exposure to the laser beam during evaporation and destruction of prostate tumor tissue is accompanied by the restoration of the structure of regional lymph nodes, which is an indicator of the activation of the local immune response, which is of important prognostic value in the postoperative period and at the stage of healing of the operating wound.

\section{REFERENCES}

1. Baldueva I. A. Immunological features of tumors and relationship of the organism with melanoma // Practical Oncology. -2001 . - v. 8, - №4. - p. $37-41$.

2. Baryshnikov A. YU., the Relationship of the tumor and the immune system //Practical Oncology. - 2003. - v. 4, №. 3. p. 127-130

3. Borodin Yu. I. Lymph node as a marker of environmental pressure on the biological system // Bulletin SB RAMS. - 1993. - №. 2. p. 5-9.

4. BORODIN Y.I., LOMSHAKOV A.A., Astashov V.V., KAzAKOV O.V., MaYOROV A.P., LARIONOV P.M. Thymus in experimental carcinogenesis of the prostate gland// Bulletin of experimental biology and medicine. - $2014-$ v.157 - №6 - p.724-727, doi: 10.1007/ s10517-014-2652-4

5. Fuchs B., Berlien H.P., Philipp C. Lasers in medicine Review / Z. Arztl. Fortbild Qualitatssich. - 1999 - Jun; 93 - №4 - p. 259-266. PMID: 10432569

6. Lomshakov A.A., Astashov V.V., KozlOV V.I., RYzHAKIN S.M., Uloga M.V., MedYANTSEVA D.A. Morphological study of lymphoid organs at early stages of experimental prostate carcinogenesis// Urologiia - 2019 - №3 - p.89-94 PMID: 31356019

7. Sarkisov D. S., Perov Yu. L. Microscopic technique: Leadership / M.: Medicine, - 1996. $544 \mathrm{p}$. 